PROCEEDINGS OF THE

AMERICAN MATHEMATICAL SOCIETY

Volume 127, Number 1, January 1999, Pages 309-314

S 0002-9939(99)04871-6

\title{
MUTATIONS OF LINKS IN GENUS 2 HANDLEBODIES
}

\author{
D. COOPER AND W. B. R. LICKORISH
}

(Communicated by Ronald A. Fintushel)

\begin{abstract}
A short proof is given to show that a link in the 3-sphere and any link related to it by genus 2 mutation have the same Alexander polynomial. This verifies a deduction from the solution to the Melvin-Morton conjecture. The proof here extends to show that the link signatures are likewise the same and that these results extend to links in a homology 3-sphere.
\end{abstract}

\section{INTRODUCTION}

Suppose $L$ is an oriented link in a genus 2 handlebody $H$ that is contained, in some arbitrary (complicated) way, in $S^{3}$. Let $\rho$ be the involution of $H$ depicted abstractly in Figure 1 as a $\pi$-rotation about the axis shown. The pair of links $L$ and $\rho L$ is said to be related by a genus 2 mutation. The first purpose of this note is to prove, by means of long established techniques of classical knot theory, that $L$ and $\rho L$ always have the same Alexander polynomial. As described briefly below, this actual result for knots can also be deduced from the recent solution to a conjecture, of P. M. Melvin and H. R. Morton, that posed a problem in the realm of Vassiliev invariants. It is impressive that this simple result, readily expressible in the language of the classical knot theory that predates the Jones polynomial, should have emerged from the technicalities of Vassiliev invariants. It may be the first such new result to arrive in this way. However, the method of proof used here depends only on elementary homology theory and so the result extends at once to give a new result for links in a homology 3 -sphere. Furthermore, as the proof produces Seifert matrices for $L$ and $\rho L$, it is simple to deduce at the same time that these links have the same classical signature $[6]$ and the same $\omega$-signatures [9]. These latter results are thus inspired by, but not directly deducible from, the resolution of the Melvin-Morton conjecture. It is interesting to note that it has been shown in [2] and [8] that the signature of a knot is not a Vassiliev invariant.

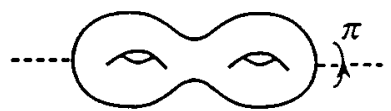

FiguRE 1

Received by the editors May 13, 1997.

1991 Mathematics Subject Classification. Primary 57M25; Secondary 81T99, 81R50.

Key words and phrases. Alexander polynomial, knot signature, knot mutation, Jones polynomial, Melvin-Morton conjecture.

This research was supported in part by N.S.F. grants DMS9504438 and DMS9510505.

(c)1999 American Mathematical Society 
The Melvin-Morton conjecture postulated a remarkable correlation between a sequence of Jones polynomials of a classical knot and the Alexander polynomial of that knot. The conjecture was proved by D. Bar-Natan and S. Garoufalidis in [1]. The background to the conjecture appears in [4] and heuristic evidence in its favour (using a path integral approach) was given by L. Rozansky [7]. The conjecture asserted that it was possible to calculate, with a specific formula quoted in [1], each coefficient in the Alexander polynomial of a knot from knowledge of all the coefficients in all the coloured Jones polynomials of the knot (with zero framing). These coloured Jones polynomials can be regarded as generalisations of the Jones polynomial invariant to a framed knot equipped with a sequence of irreducible representations of $\mathrm{SU}(2)$. Alternatively, interpreting the Jones invariant by means of the skein theory of the Kauffman bracket, the coloured invariants are the evaluations of the knot decorated by Chebyshev polynomials in the generator of the skein of the solid torus. The proof given in [1] is an intricate and skillful deployment of Vassiliev invariants analysed by means of arguments with chord diagrams and weight systems. Indeed, it was in the world of these ideas that the Melvin-Morton conjecture was originally conceived.

A consequence of the solution to the Melvin-Morton conjecture is, of course, that if two knots have the whole sequence of coloured Jones polynomials identical, then their Alexander polynomials are also the same. What examples are there of pairs of knots that satisfy this hypothesis? Any two knots related by a classical mutation (in the sense of J. H. Conway) give a first example. It is a standard result that two such knots have the same Alexander polynomial; a proof can be given using Conway's skein theory which immediately shows that the knots also have the same HOMFLY polynomial. However a second example is provided by knots $K$ and $\rho K$ that are related by a genus 2 mutation as described above. They have all coloured Jones invariants the same. For that, an easy proof is simply to note that the Kauffman bracket skein, of a genus 2 handlebody $H$, has a base consisting of certain sets of disjoint, closed, unoriented, framed curves in $H$, each such set being invariant under $\rho$. (This idea has also been employed by A. Kawauchi in [3].) It thus follows from the above mentioned results that $K$ and $\rho K$ have the same Alexander polynomial.

It is fairly easy to see that if two knots differ by a classical Conway mutation, then they differ by a sequence of at most two of the genus 2 mutations described above. In particular, in the famous example of two eleven crossing knots, the Conway knot and the Kinoshita-Terasaka knot, the two knots are related by a single genus 2 mutation. This implies that a satellite of one of these knots is related by genus 2 mutation to the same satellite of the other. Computer calculations of Morton and H. B. Short (see [5]) have shown that a certain cabling of the Conway knot has its HOMFLY polynomial different from that of the same cabling of the Kinoshita-Terasaka knot. Thus a genus 2 mutation can indeed change a knot and, furthermore, a genus 2 mutation is not always just a sequence of classical mutations, for the latter preserve HOMFLY polynomials. In addition, this example makes it hard to see how skein theory could be used to show that $K$ and $\rho K$ always have the same Conway polynomial. They do have the same Conway polynomial, as that is but a normalisation of the Alexander polynomial. However, Conway skein theory is very similar to HOMFLY skein theory. 


\section{Preliminary Results}

There follow here four elementary results that will be used later.

Lemma 1. Suppose that $H$ is a handlebody of genus $g$ contained in $S^{3}$ and let $X$ be the closure of $S^{3}-H$. The inclusion maps of $\partial H$ into $H$ and $X$ induce an isomorphism $H_{1}(\partial H) \rightarrow H_{1}(H) \oplus H_{1}(X)$.

Proof. The Mayer-Vietoris exact homology sequence applied to $S^{3}$ expressed as $H \cup X$ provides this isomorphism at once.

This result implies that each of $H_{1}(H)$ and $H_{1}(X)$ is a copy of $\mathbb{Z}^{g}$. Furthermore, letting $i: \partial H \rightarrow X$ be the inclusion map, it follows that the kernel of $i_{\star}: H_{1}(\partial H) \rightarrow$ $H_{1}(X)$ is a direct summand of $H_{1}(\partial H)$ and that this kernel maps isomorphically onto $H_{1}(H)$.

Lemma 2. Suppose that a collection of disjoint, oriented, simple, closed curves, contained in the boundary of an orientable 3-manifold $M$, represents the zero element of $H_{1}(M)$. Then those curves form the boundary of some oriented surface properly embedded in $M$.

This is a standard result that can, for example, be proved by means of transversality.

Now let $H$ be a genus 2 handlebody and $\rho$ be the homeomorphism of $H$ to itself given by rotation through $\pi$ about the axis shown in Figure 1. In that diagram $H$ is shown in a standard position in $\mathbb{R}^{3}$. If $H$ is embedded in some other way, then it is the conjugate, by the embedding, of this rotation that is to be called $\rho$.

Lemma 3. If $C$ is any simple closed curve in $\partial H$, then $C$ is isotopic to a curve $\widetilde{C}$ that is fixed, as a set, by $\rho$. If $C$ separates $\partial H$, then $\rho$ preserves the orientation of $\widetilde{C}$, otherwise $\rho$ reverses the orientation of $\widetilde{C}$.

Proof. It is a classical result that $\rho \mid \partial H$ represents the only non-trivial element in the centre of the mapping class group of $\partial H$. In fact, any orientation preserving automorphism $\phi$ of $\partial H$ is isotopic to some $\widetilde{\phi}$ that commutes with $\rho$. If $C$ separates $\partial H$ there is some such $\phi$ for which $\phi C$ is equal to a "standard curve" $C_{1}$ that separates and is preserved, together with its orientation, by $\rho$. Then $\tilde{\phi}^{-1} C_{1}$ is isotopic to $C$ and is preserved by $\rho$. Similar remarks apply when $C$ does not separate.

Suppose that, with entries from some commutative ring, $M$ is an $r \times r$ matrix and $N$ is an $s \times s$ matrix and that $M_{r, r}=N_{1,1}$. Let $M \oplus_{1} N$ be the $(r+s-1) \times(r+s-1)$ matrix that has $M$ for its first $r \times r$ terms, $N$ for its final $s \times s$ terms and zeros elsewhere (see Figure 2). Let $\widetilde{M}$ and $\widetilde{N}$ be obtained by deleting the last row and column from $M$ and the first row and column from $N$.

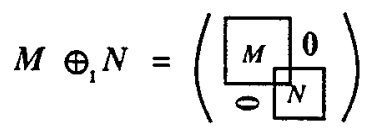

FigURE 2 
Lemma 4. With the above notation,

$$
\operatorname{det}\left(M \oplus_{1} N\right)=\operatorname{det} \widetilde{M} \operatorname{det} N+\operatorname{det} M \operatorname{det} \widetilde{N}-M_{r, r} \operatorname{det} \widetilde{M} \operatorname{det} \widetilde{N} .
$$

Proof. This follows at once by expanding the determinant by the $r^{\text {th }}$ row.

Note that it follows at once from this result that $\operatorname{det}\left(M \oplus_{1} N\right)=\operatorname{det}\left(M \oplus_{1} N^{\tau}\right)$, where $N^{\tau}$ denotes the transpose of the matrix $N$.

\section{SEIFERT MATRICES}

It will now be shown that there are closely related Seifert matrices for a link $L$ and any genus 2 mutation of $L$.

Theorem 5. Let $L$ be an oriented link contained in a genus 2 handlebody $H$ which is contained in $S^{3}$. Let $\rho: H \rightarrow H$ be the involution that, when restricted to $\partial H$, represents the non-trivial element of the centre of the mapping class group of $\partial H$. Then $L$ and $\rho L$ have Seifert matrices of the form

$$
\left(\begin{array}{cc}
A & B^{\tau} \\
B & C
\end{array}\right) \text { and }\left(\begin{array}{ll}
A & B^{\tau} \\
B & C^{\tau}
\end{array}\right)
$$

respectively, where the blocks are matrices of integers, $A$ and $C$ are square and $B$ is of the form $(0 \mid \boldsymbol{b})$ for some single column $\boldsymbol{b}$.

Proof. Let $X$ be the closure of $S^{3}-H$. Suppose that $F_{0}$ is, for the link $L$ in $S^{3}$, a Seifert surface which intersects $\partial H$ transversely. The element of $H_{1}(\partial H)$ represented by $F_{0} \cap \partial H$ can, of course, be expressed as $n[\alpha]$, for some $n \in \mathbb{Z}$ with $n \geq 0$, where $[\alpha]$ is an indivisible element of $H_{1}(\partial H)$. Now, $n[\alpha]$ belongs to the kernel of $i_{\star}: H_{1}(\partial H) \rightarrow H_{1}(X)$ where $i$ is the inclusion map. By Lemma 1 this implies that, for $n \neq 0,[\alpha]$ belongs to the kernel of $i_{\star}$ (as this kernel is a direct summand). Thus $[\alpha]$ maps by inclusion to an element of a base of $H_{1}(H)$. This indivisible element $[\alpha]$ can be represented by an essential oriented simple closed curve $\alpha$ contained in $\partial H$. By Lemma 2, applied to $H$ less a neighbourhood of $L$, there is a connected orientable surface $F_{1}$ contained in $H$ such that $\partial F_{1}$ is the union of $L$ and $n$ parallel copies of $\alpha$. Again by Lemma 2, $\alpha$ bounds a connected orientable surface $F_{2}$ embedded in $X$. Let $F=F_{1} \cup n F_{2}$ where $n F_{2}$ is $n$ parallel copies of $F_{2}$. This $F$ is the required Seifert surface for $L$.

A base for $H_{1}(F)$ is represented by the oriented curves $\left\{\gamma_{1}, \gamma_{2}, \ldots, \gamma_{r}\right\}$ and $\left\{\delta_{1}, \delta_{2}, \ldots, \delta_{s-1}\right\}$ where the $\left\{\gamma_{i}\right\}$ lie in $F_{1}$ and the $\left\{\delta_{i}\right\}$ represent a base of $H_{1}\left(n F_{2}\right)$. Let $\beta$ be an oriented closed curve contained in $H$ such that $[\alpha]$ and $[\beta]$ form a base for $H_{1}(H)$. After making changes to the $\left\{\gamma_{i}\right\}$ by elementary moves, it can be assumed that, in $H_{1}(H),\left[\gamma_{i}\right]=m_{i}[\alpha]$ for $i=1,2, \ldots, r-1$ with $m_{i} \in \mathbb{Z}$, and $\left[\gamma_{r}\right]=m_{r}[\alpha]+n_{r}[\beta]$. With respect to this base, the Seifert matrix is of the form $\left(\begin{array}{cc}A & B^{\tau} \\ B & C\end{array}\right)$, where the blocks, defined in terms of linking numbers, are given by $A_{i, j}=\operatorname{lk}\left(\gamma_{i}^{-}, \gamma_{j}\right), C_{i, j}=\operatorname{lk}\left(\delta_{i}^{-}, \delta_{j}\right)$ and $B_{i, j}=\operatorname{lk}\left(\delta_{i}^{-}, \gamma_{j}\right)$. Here $\gamma_{i}^{-}$and $\delta_{i}^{-}$are curves obtained by pushing $\gamma_{i}$ and $\delta_{i}$ off $F$ in a "negative" direction that is determined by the orientation of $F$ (which is induced by that of $L$ ) and the orientation of $S^{3}$. Observe that $\operatorname{lk}\left(\delta_{i}^{-}, \gamma_{r}\right)=m_{r} \operatorname{lk}\left(\delta_{i}^{-}, \alpha\right)+n_{r} \operatorname{lk}\left(\delta_{i}^{-}, \beta\right)$. However, $\operatorname{lk}\left(\delta_{i}^{-}, \alpha\right)=0$ because $\alpha$ bounds a surface, a copy of $F_{2}$, that is disjoint from $\delta_{i}^{-}$. Hence $B_{i, j}=0$ for $j<r$ and $B_{i, r}=n_{r} \operatorname{lk}\left(\delta_{i}^{-}, \beta\right)$. This gives a Seifert matrix for $L$ of the required form. 
Now consider $\rho L$. By Lemma 3 it may be assumed that $\rho$ fixes the simple closed curve $\alpha$ as a set, but that $\rho$ reverses the orientation of $\alpha$. Thus $\rho$ maps $n$ parallel copies of oriented $\alpha$ to the same set of curves but reverses the order of the components and reverses orientations. Hence a Seifert surface $F_{\rho}$ for $\rho L$ consists of $\rho F_{1} \cup n\left(-F_{2}\right)$ where the minus sign denotes a change in orientation. For a base of $H_{1}\left(F_{\rho}\right)$ take the classes represented by $\left\{\rho \gamma_{1}, \rho \gamma_{2}, \ldots, \rho \gamma_{r}\right\} \cup\left\{-\delta_{1},-\delta_{2}, \ldots,-\delta_{s-1}\right\}$. Now, consideration of the intersection of $\gamma_{i}^{-}$with a surface spanning $\gamma_{j}$ shows that $\operatorname{lk}\left(\gamma_{i}^{-}, \gamma_{j}\right)=\operatorname{lk}\left(\rho \gamma_{i}^{-}, \rho \gamma_{j}\right)$. Because the "negative" direction for pushing a curve off $-F_{2}$ is opposite to that for $F_{2}$, calculation with respect to $F_{\rho}$ gives $\operatorname{lk}\left(-\delta_{i}^{-},-\delta_{j}\right)=C_{j, i}$. Also, $\operatorname{lk}\left(\delta_{i}^{-}, \rho \beta\right)=-\operatorname{lk}\left(\delta_{i}^{-}, \beta\right)$ because $\rho_{\star}=-1$ on $H_{1}(H)$. Thus $n_{r} \operatorname{lk}\left(-\delta_{i}^{-}, \rho \beta\right)=B_{i, r}$. Thus the Seifert matrix for $\rho L$ with respect to the above base for $H_{1}\left(F_{\rho}\right)$ is indeed $\left(\begin{array}{ll}A & B^{\tau} \\ B & C^{\tau}\end{array}\right)$.

Note that it is known that the Conway knot and the Kinoshita-Terasaka knot, which are related by a genus 2 mutation, do not have the same knot genus. Thus, in the above proof it is necessary to take some care when correlating the Seifert surfaces of $L$ and $\rho L$.

Corollary 6. The links $L$ and $\rho L$ have the same Conway-normalised Alexander polynomial.

Proof. If $S$ is a Seifert matrix for $L$, then the Conway-normalised Alexander polynomial is $\operatorname{det}\left(t^{1 / 2} S-t^{-1 / 2} S^{\tau}\right)$. Suppose $S=\left(\begin{array}{cc}A & B^{\tau} \\ B & C\end{array}\right)$ where $B=(0 \mid \boldsymbol{b})$. Then the Alexander polynomial for $L$ is the determinant of

$$
\left(\begin{array}{cc}
t^{1 / 2} A-t^{-1 / 2} A^{\tau} & \left(t^{1 / 2}-t^{-1 / 2}\right) B^{\tau} \\
\left(t^{1 / 2}-t^{-1 / 2}\right) B & t^{1 / 2} C-t^{-1 / 2} C^{\tau}
\end{array}\right)
$$

The Alexander polynomial of $\rho L$ is, by the theorem, the determinant of an identical matrix except that the bottom right block is transposed to become $\left(t^{1 / 2} C^{\tau}-\right.$ $\left.t^{-1 / 2} C\right)$. However, because $B=(0 \mid \boldsymbol{b})$, these determinants are the same, by Lemma 4 (substituting $\left(t^{1 / 2} A-t^{-1 / 2} A^{\tau}\right)$ for $M$ ).

Corollary 7. Let $L$ be an oriented link contained in a genus 2 handlebody $H$ that is contained in a homology 3-sphere $M^{3}$. Then $L$ and $\rho L$ have the same Alexander polynomials.

Proof. This follows by exactly the same method as that given for $S^{3}$ as only homology techniques were used.

Corollary 8. Let $L$ be an oriented link contained in a genus 2 handlebody $H$ that is contained in a homology 3-sphere $M^{3}$. Then $L$ and $\rho L$ have all the same $\omega$ signatures.

Proof. If $S$ is a Seifert matrix of $L$ and $\omega$ is a complex number of unit modulus, $\omega \neq 1$, then the $\omega$-signature of $L$ is the signature of the Hermitian matrix $(1-\omega) S+(1-\bar{\omega}) S^{\tau}$. Take for $S$ the matrix $\left(\begin{array}{cc}A & B^{\tau} \\ B & C\end{array}\right)$ and let $S_{\rho}$ be $\left(\begin{array}{ll}A & B^{\tau} \\ B & C^{\tau}\end{array}\right)$ where $B=(0 \mid \boldsymbol{b})$. Now, any Hermitian matrix has real eigenvalues, and its signature is the difference between the number of positive eigenvalues and the number of negative eigenvalues. The matrix $(1-\omega) S_{\rho}+(1-\bar{\omega}) S_{\rho}^{\tau}$ can be obtained from $(1-\omega) S+(1-\bar{\omega}) S^{\tau}$ by transposing the block consisting of the final $s$ rows and 
columns. Thus these two matrices have, by Lemma 4, the same characteristic polynomial; hence they have the same eigenvalues and the same signature.

\section{REFERENCES}

1. D.Bar-Natan and S.Garoufalidis, On the Melvin-Morton-Rozansky conjecture, Invent. Math. 125 (1996), 103-133. MR 97i:57004

2. J.Dean, Many classical knot invariants are not Vassiliev invariants, J. Knot Theory and its Ramifications 3 (1994), 7-10. MR 94k:57008

3. A.Kawauchi, Topological imitation, mutation and quantum SU(2) invariant, J. Knot Theory and its Ramifications 3 (1994), 25-39. MR 95a:57025

4. P.M.Melvin and H.R.Morton, The coloured Jones function, Comm. Math. Phys. 169 (1995), 501-520. MR 96g:57012

5. H.R.Morton and H.B.Short, Calculating the 2-variable polynomial for knots presented as closed braids, J. Algorithms 11 (1990), 117-131. MR 91f:57004

6. K.Murasugi, On a certain numerical invariant of link types, Trans. Amer. Math. Soc. 117 (1965), 387-422. MR 30:1506

7. L.Rozansky, A contribution of the trivial connection to the Jones polynomial and Witten's invariant of $3 d$ manifolds, I and II, Comm. Math. Phys. 2175 (1996), 275-296, 297-318. MR 97e:57038

8. R.Trapp, Twist sequences and Vassiliev invariants, J. Knot Theory and its Ramifications 3 (1994), 391-405. MR 95h:57012

9. A.G.Tristram, Some cobordism invariants for links, Proc. Cambridge Philos. Soc. 66 (1969), 251-264. MR 40:2104

Department of Mathematics, University of California at Santa Barbara, Santa BarBARA, CALIFORNIA 93106

E-mail address: cooper@math.ucsb.edu

Department of Pure Mathematics and Mathematical Statistics, University of CamBridge, 16 Mill Lane, Cambridge, CB2 1SB, United Kingdom

E-mail address: wbrl@dpmms.cam.ac.uk 